\title{
Investigation on the Applicability of the Time Domain Analysis of Discharges in Gases for the Defect Identification at AC Voltage
}

\author{
Ulrich Lühring, Daniel Wienold, Frank Jenau
}

\begin{abstract}
The partial discharge diagnosis is an established instrument for the condition assessment of high voltage insulations and equipment. Under AC voltage stress the phase resolved pattern is of great significance in order to become aware of the type of fault. As a result of the inapplicability for DC voltage stress, approaches for alternative interpretation techniques such as the time domain analysis of partial discharges were identified in recent investigations. In these different types of fault are taken into account as well as different insulating media. The purpose of this paper is to investigate whether an analysis of the pulse shape is also applicable for the defect identification under AC voltage stress. By focussing on gaseous insulating media, contact noise and surface discharges are emulated in ambient air, whereas corona discharges are emulated in ambient air and oxygen. A method for analysing discharges, occurring in the negative and the positive half-wave of the test voltage, is proposed and discussed.
\end{abstract}

Index Terms-AC voltage, contact noise, corona discharge, pulse shape, surface discharge, time domain analysis

\section{INTRODUCTION}

$\mathrm{P}$ ARTIAL discharges (PD) are a result of local electrical stress concentration and defined as localized discharges, partially bridging the insulation between conductors [1]. Due to the fact that PD can indicate serious defects at an early stage, the PD diagnosis is a valuable method for the fault detection. Under AC voltage stress the PD diagnosis is rather an established tool to identify the location and the type of fault [2]. The latter is possible because of different involved processes going along with the type of fault and the associated influence on the appearance of PD. To draw conclusions regarding the type of fault through measuring and interpreting PD, the phase resolved pattern is of great practical importance at $\mathrm{AC}$ voltage. In this approach, the superimposition of the discharge pulses and the phase angle of the test voltage is analysed. A transfer of this analysis method to DC voltage stress is inadequate. For that reason and the fact that $\mathrm{DC}$ transmission systems are increasingly important, it is necessary to develop and evaluate alternative and suitable basic approaches.

With regard to partial discharges occurring under DC voltage stress the discharge magnitude, the time lag between PD impulses [3] and the pulse shape of the discharge current are values that can be obtained from electrical measurements. This paper focuses on the pulse shape, which is characterised by a steep ascent and a slower descent. An analysis of the pulse shape enables to draw conclusions regarding the discharge mechanism if the discharge current is not distorted. Therefore an optimised measuring circuit and a measuring instrument with a single shot bandwidth of at least $1 \mathrm{GHz}$ is required [4].

A model-based description of the pulse shape characteristic is presented by Loeb [5]. Referring to this, Morrow calculates the development of the PD process theoretically for negative corona discharges in oxygen [6]. One of the first extensive experimental investigations on the pulse shape of corona discharges is presented by Scott [7]. It confirms Morrow's theoretical calculations in several respects. Subsequent investigations are primarily carried out with the purpose of gaining more detailed information about the physical processes of partial discharges. Furthermore a link between the pulse shape and the ageing condition of solid insulating media is established. According to this the relationship between the pulse shape and influencing factors such as the pressure [7]-[9], the gas mixture [10], the voltage level [7] and the stress duration [4], [11] are investigated as well as the dependence of the size and position of voids in solid insulating media [12]-[13]. The abovementioned investigations are carried out by focussing on one type of fault.

Comparative investigations on the pulse shape of partial discharge occurring with different type of faults are presented amongst others in [14]-[16]. Captured pulse shapes are shown, but a qualitative and statistical comparison of pulse shape

This manuscript was submitted on October 24, 2016 and revised on December 23, 2016 as an extended version of the paper "Applicability of coefficients for the defect identification of partial discharges in air under $A C$ voltage stress based on the pulse shape" presented at the $16^{\text {th }}$ International Conference on Environment and Electrical Engineering, Florence/Italy, June 2016.

Ulrich Lühring, Daniel Wienold and Frank Jenau are with the Institute of High Voltage Engineering, TU Dortmund University, Dortmund, Germany (e-mail: ulrich.luehring@ tu-dortmund.de).

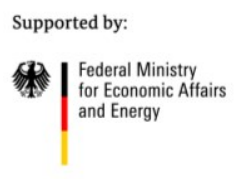

This work has been funded by the German Federal Ministry for Economic Affairs and Energy (BMWi) as a part of the $\mathrm{E}^{2} \mathrm{HGÜ} \mathrm{project} \mathrm{under} \mathrm{grant}$ number 03ET7514. 
parameters is not conducted. Nevertheless it can be concluded that the time domain analysis of partial discharges seems to be suited for distinguishing different types of fault from each other. For partial discharges occurring under DC voltage stress this is proven in [17]-[21]. Whereas the suitability for an identification of different types of fault appearing in the same insulating medium is shown in [18], [19] points out that an analysis of the pulse shape is suited to differentiate between varying insulating media as well. Primary pulse shape parameters such as the rise time, the pulse width and the fall time are factored in those investigations as well as secondary pulse shape parameters such as the scatter and the symmetry of the distribution [20]. In [21] it is shown that the primary parameters and the coefficient of variation, as a tool to evaluate the scatter, are especially suited for the defect identification of PD under DC voltage stress.

Due to these findings the applicability of the time domain analysis of discharges to identify the type of fault and to differentiate between varying gaseous insulating media under $\mathrm{AC}$ voltage stress is investigated in this paper. Focussing on external PD and internal disturbance sources three typical types of fault, which are confirmed by displaying the phase resolved pattern, are emulated in ambient air and partially in oxygen. The pulse shapes occurring in the positive and the negative halfwave of the test voltage are captured and analysed.

\section{MEASUREMENT SETUP}

\section{A. Measuring cell}

Resonance effects as well as reflection and refraction processes can lead to distortions of the pulse shape. As a result, an accurate measurement of the pulse shape requires a measuring cell optimized from a high frequency and high voltage point of view. The sectional view of an optimized broadband measuring cell is shown in Fig. 1. This measuring cell complies with the one presented and already used in [22] respectively [18]-[21].

Using a constant line impedance of $50 \Omega$, the conical

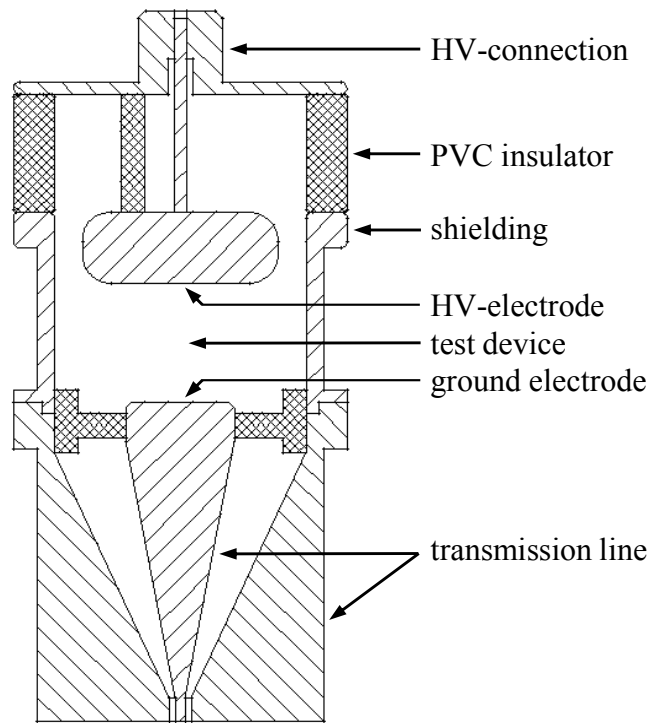

Fig. 1. Sectional view of the measuring cell optimised from a high frequency and high voltage point of view; following [22]. transmission line enables a decoupling of the discharge impulse current from the test object to the measuring instrument devoid of reflections and refractions. The shielded low-inductive designed measuring cell reduces the disturbance impact of external sources. In [22] and [19] it is proven that the bandwidth (BW) of the measuring cell is $\mathrm{BW}>1 \mathrm{GHz}$ and that an accurate measurement of the pulse shape of discharges is possible.

\section{B. Measuring Circuit}

The measuring cell is placed in a basic PD measuring circuit according to IEC 60270. As shown in Fig. 2, besides the measuring cell with the test device (DUT), the measuring circuit consists, of a coupling capacitor (Omicron MCC 210-L) $C_{\mathrm{c}}$, a decoupling impedance $Z_{\mathrm{d}}$ and a measuring impedance $Z_{\mathrm{m} 1}$ respectively $Z_{\mathrm{m} 2}$. Whereas $Z_{\mathrm{d}}$ assures a decoupling of the discharge current circuit from the high voltage power supply $U_{0}$, the measuring impedance allows a measurement of the discharge impulse current $i_{\mathrm{PD}}$. To record the phase resolved pattern a PD measuring system (Omicron MPD 600 with CPL 542) with an external quadripole is set as $Z_{\mathrm{m} 1}$ in series to $C_{\mathrm{c}}$. In contrary to that the input resistor of an oscilloscope (LeCroy Waverunner 640Zi) with a sampling rate of $20 \mathrm{GS} / \mathrm{s}$ and a single shot bandwidth of $4 \mathrm{GHz}$ is set as $\mathrm{Zm} 2$ in series to the DUT for capturing the pulse shapes. The maximum bandwidth of the entire measuring circuit is bounded by the measuring cell. According to [4] the required bandwidth is at least $1 \mathrm{GHz}$ which enables an accurate measurement of the pulse shape.

\section{TEST DEVICES}

Typical types of PD are internal discharges occurring in the case of flaws in insulating liquids or solid dielectrics and external discharges such as corona discharges and surface discharges [2]. It has to be taken into account that the measurement and interpretation of PD can be hampered significantly by disturbance sources. Whereas external disturbance sources, which are independent of the measuring circuit, can be regarded as uncritical up to a certain external noise level, voltage dependent internal disturbance sources must be considered separately with regard to the interpretation of the appearance of partial discharges [23]. A typical internal disturbance source is contact noise, which occurs for example in the case of insufficient conductive connection [24]. Taking

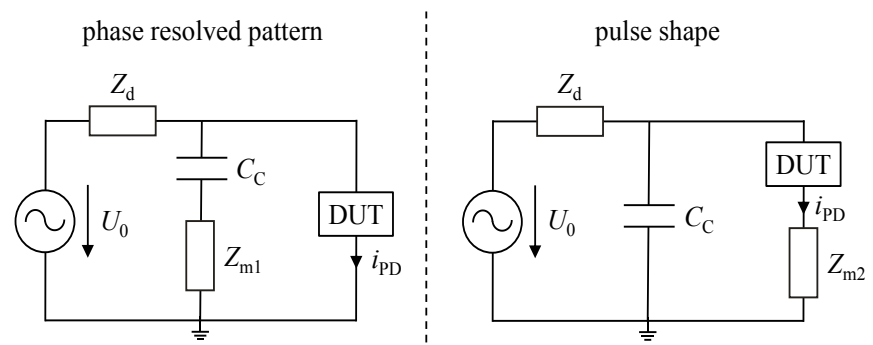

Fig. 2. Basic PD measuring circuit with $\mathrm{AC}$ source $U_{0}$, decoupling impedance $Z_{\mathrm{d}}$, coupling capacitor $C_{\mathrm{c}}$, measuring cell with test device DUT and measuring impedance $Z_{\mathrm{m} 1}$ respectively $Z_{\mathrm{m} 2}$ to capture the phase resolved pattern (left) and the pulse shape (right). 
the latter and the focus on PD in gases into consideration, the applicability of the time domain analysis to identify the type of fault under $\mathrm{AC}$ voltage stress is investigated by recreating and analysing external discharges as well as contact noise. The different test devices are installed independently of one another in the measuring cell and are described in the following.

\section{A. Contact noise}

Leaving an air gap of $2 \mathrm{~mm}$ between the HV-connection and the cylindrical electrode causes an inadequate conductive connection, which emulates contact noise.

\section{B. Surface discharge}

To emulate surface discharges a PVC plate with a diameter of $30 \mathrm{~mm}$ and a thickness of $5 \mathrm{~mm}$ is placed centred between the ground electrode and a steel sphere with a diameter of $7 \mathrm{~mm}$. The steel sphere appears as high voltage electrode.

\section{Corona discharges}

To generate corona discharges a needle-to-plate setup is used. A needle with a tip radius of approximately $75 \mu \mathrm{m}$ is placed on the high voltage cylindrical electrode. The air clearance between the needle tip and the ground electrode is set to $35 \mathrm{~mm}$.

\section{MEASUREMENTS AND ANALYSIS}

All measurements are performed under ambient atmosphere and $10 \%$ above the discharge inception voltage, which is determined in advance. Due to the polarity effect, the discharge inception voltage may depend on the polarity of the test voltage. This can lead to the necessity of two different test voltages for the same test device to capture discharges in the positive and the negative half-wave under comparable test conditions.

As shown in Fig. 3 at least two different test series are carried out with each of the three test devices. Under assignment of ambient air as insulating medium the first test series are carried out to confirm the type of fault. For this the PD measuring system is used to display the phase resolved pattern. By using the oscilloscope, the purpose of the following test series is the capture of the pulse shapes occurring in the negative and the positive half-wave of the test voltage. These measurements are carried out with the insulating medium ambient air and partially with the insulating medium oxygen. To investigate whether the time domain analysis is applicable to differentiate between different types of faults, the former are performed with all of the three test devices. The execution of the latter is restricted to the needle-to-plane setup, whereby the purpose of this measurements is to examine exemplarily whether an analysis of the pulse shape is suited to differentiate between varying insulating media as well.

Concerning the pulse shape measurements 500 sequent pulse shapes of discharges are captured in every measurement after a stress duration of a few minutes. In Fig. 4 the pulse shape of a corona discharge is shown exemplarily with voltage values normalised to the maximum. Relating to [5] the steep ascent of a negative corona discharge is caused by avalanches near the cathode which are induced by free electrons with a high

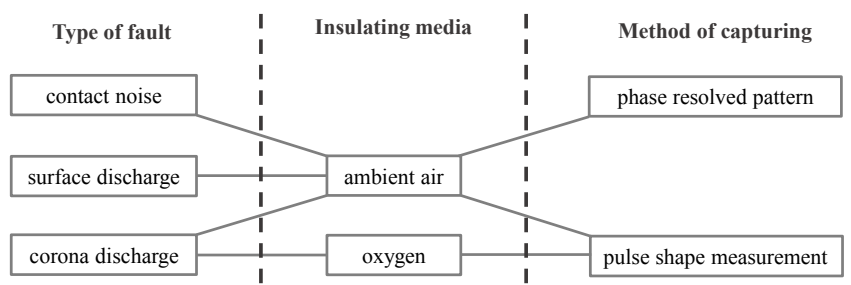

Fig. 3. Overview of the measurements carried out.

mobility. The generation of secondary electrons by ionization processes goes along with the emergence of positive ions. As a result of the lower mobility of the positive ions a positive space charge is formed near the cathode. The effective field strength behind this space charge is reduced, so that the number of ionization processes decreases in this area. Furthermore the free electrons in this area are converted to negative ions by attachment which leads to a descent of the discharge current. As shown in Fig. 4 the pulse shapes are characterised by determining the $20 \%$ to $80 \%$ rise time, the $50 \%$ to $50 \%$ pulse width and the $80 \%$ to $20 \%$ fall time as primary parameters

For the comparative presentation box plots are used, which gain a detailed overview of the location and the distribution of the determined values. As pictured in Fig. 5, this form of presentations divides the entire range of values graphically into four subsets. $50 \%$ of the values are lower and $50 \%$ of the values are higher than the median, so that the centre of the distribution is characterised by the median. The $25 \%$ quartile and the $75 \%$ quartile are set as the boundaries of a box, whose length is equal to the interquartile range (IQR) and which contains $50 \%$ of all determined values. The lowest $25 \%$ and the highest $25 \%$ of the values are connected to the box by lines, which are designated as whiskers. Regarding this it has to be noted that the maximum length of the whiskers is set to 1.5 times of the IQR. If the distance between the box and the lowest respectively the highest value is smaller than the maximum whisker length, the whisker ends at this value. Values having a greater distance to the box are characterised by a plus symbol. The notched form of presentation gives additional information about the confidence interval of the median. If the notches for different boxes do not overlap, a strong differentiation of the data values can be assumed [25].

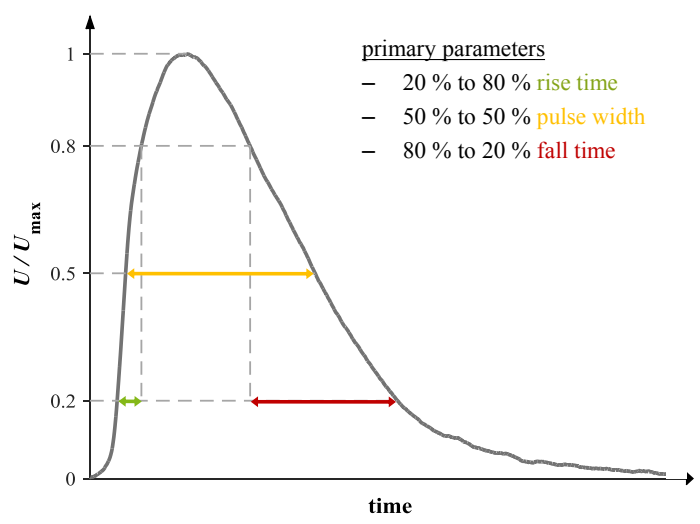

Fig. 4. Pulse shape of a corona discharge and characterising primary parameters.

In addition to the mentioned primary parameters the 


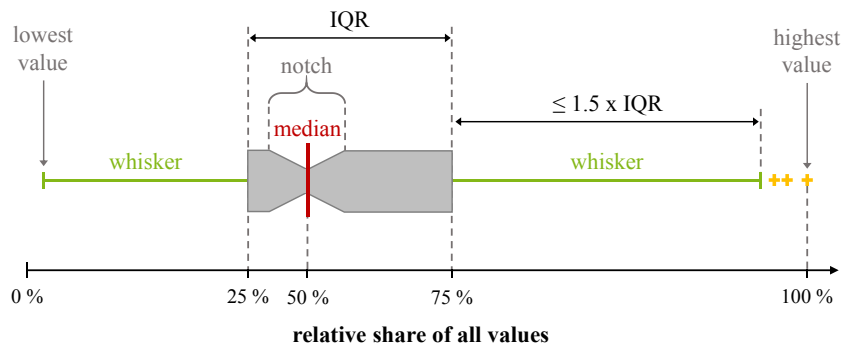

Fig. 5. Structure of a box plot.

coefficient of variation, which is the quotient of the standard deviation and the arithmetic mean, is taken into account as a secondary parameter to evaluate the scatter.

\section{RESULTS AND DISCUSSION}

\section{A. Phase resolved pattern}

The phase resolved patterns of the three test devices, which are illustrated in Fig. 6, point out differences in the pattern of the discharges in ambient air as well as in the apparent charge level. With approximately $53 \mathrm{nC}$, the contact noise shows the greatest value for the apparent charges which occur in the area of the maximum voltage change. Surface discharges occur in the area between zero voltage and crest value and the apparent charge reaches values up to $6 \mathrm{nC}$. Corona discharges embrace a range of $75 \mathrm{pC}$ to $190 \mathrm{pC}$ in the positive half-wave and a range of $15 \mathrm{pC}$ to $25 \mathrm{pC}$ in the negative half-wave. They are observed within the realm of the crest value of the test voltage.

The illustration of the phase resolved pattern meets the expectations stated in [2] and [23]. According to this, the types of fault are considered to be confirmed.

\section{B. Pulse shape measurements}

\section{1) Type of fault}

The box plots for the resulting primary parameters rise time, pulse width and fall time of contact noise, surface discharges and corona discharges in ambient air occurring in the positive and the negative half-wave of the test voltage are depicted in Fig. 7. The primary parameters of the pulse shapes of corona discharges and surface discharges correspond with the ones in [26] and [27]. Consequently, also the primary parameters of the contact noise are presumed as correct.

Fig. 7 illustrates that the medians for the corona discharges partly differ from those for the surface discharges and the contact noise. A comparison of the location and the range of the confidence intervals, broken down according to the polarity of the discharges, clarifies that an overlapping is not determined with exception of those for the rise time of positive contact noise and corona discharges. A clear distinction of these types of fault can be applied under the consideration of the values for the pulse width and the fall time. With regard to the chosen test conditions, this leads to the conclusion that the time domain analysis is appropriate to differentiate between varying types of fault.

Furthermore, Fig. 7 shows that the scatter of the determined values depends on the primary parameter and the type of fault.
Referring to all considered primary parameters and types of fault in ambient air, Fig. 8 pictures that the coefficients of variation are in the range between 0.05 and 0.67 . Corresponding to the distribution of the values for the primary parameters, the values for the coefficient of variation are for corona discharges predominantly lower than for surface discharges and contact noise. A comparison of the values points out that the coefficient of variation is especially suited for the defect identification of discharges in air.
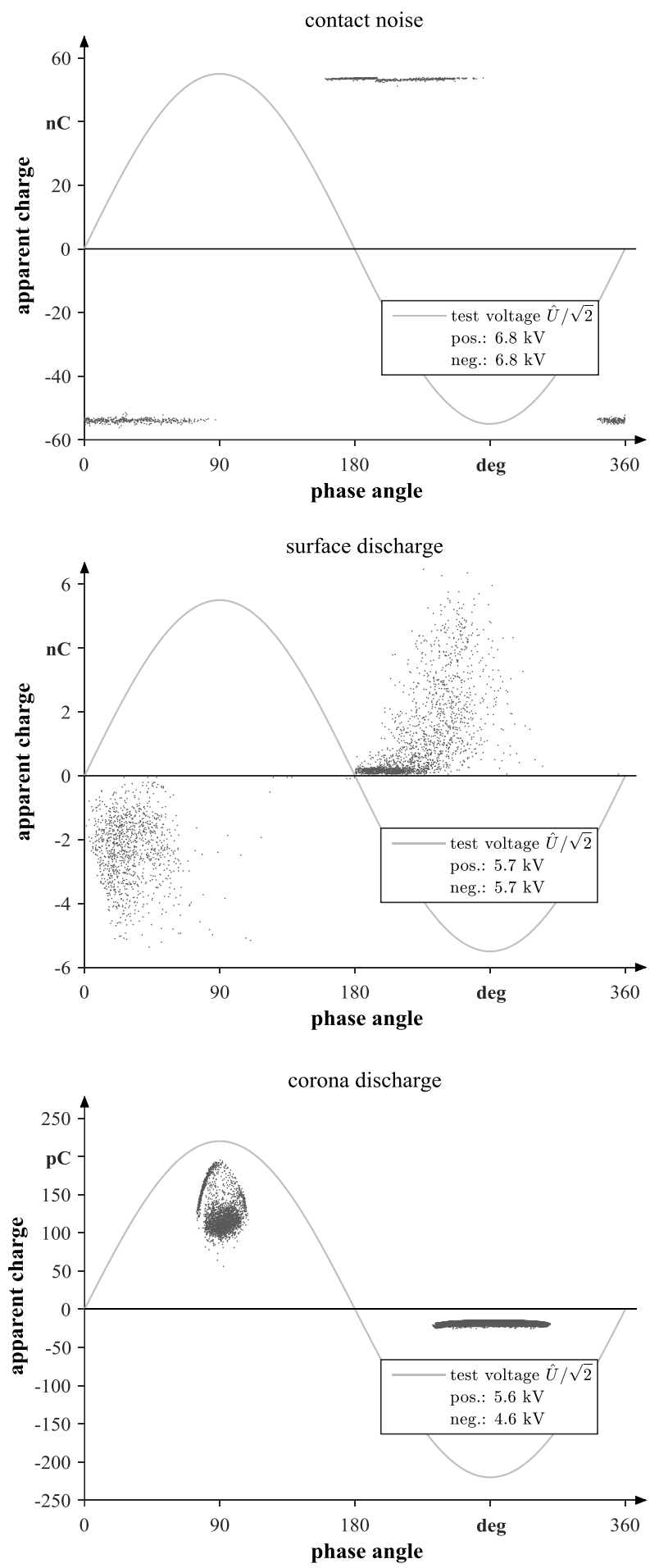

Fig. 6. Phase resolved pattern of the contact noise (top), the surface discharge (centre) and the corona discharge (bottom) in ambient air with indication of the positive and negative test voltage for each test device. 

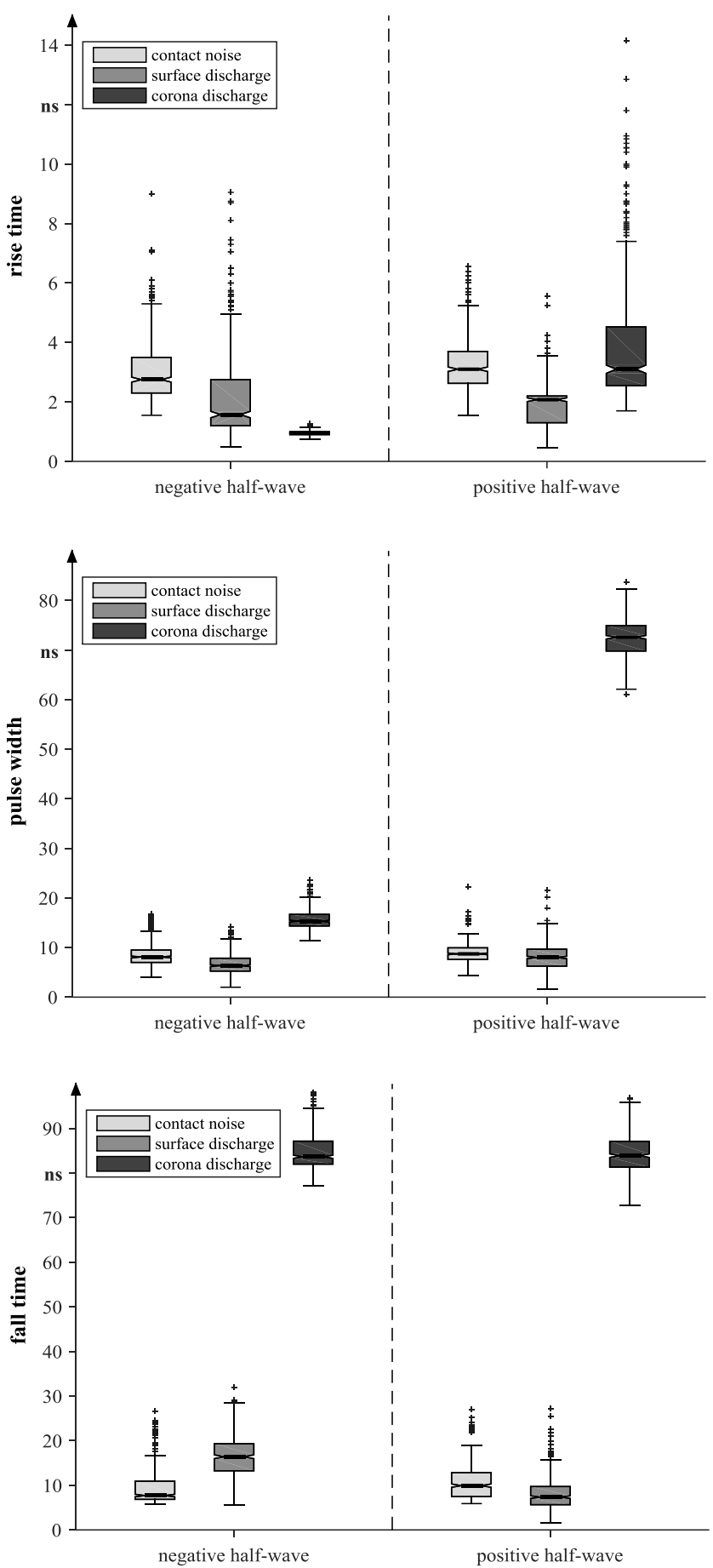

Fig. 7. Box plots for the pulse shape parameters rise time (top), pulse width (centre) and fall time (bottom) of contact noise, surface discharges and corona discharges in ambient air occurring in the negative (left) and the positive (right) half-wave of the test voltage.

\section{2) Insulating media}

The box plots for the resulting primary parameters of corona discharges in ambient air and oxygen occurring in the positive and the negative half-wave of the test voltage are shown in Fig. 9. The associated coefficients of variation are depicted in Fig. 10. The pulse shape parameters increase starting from the rise time to the fall time. Moreover, it is evident that the pulse
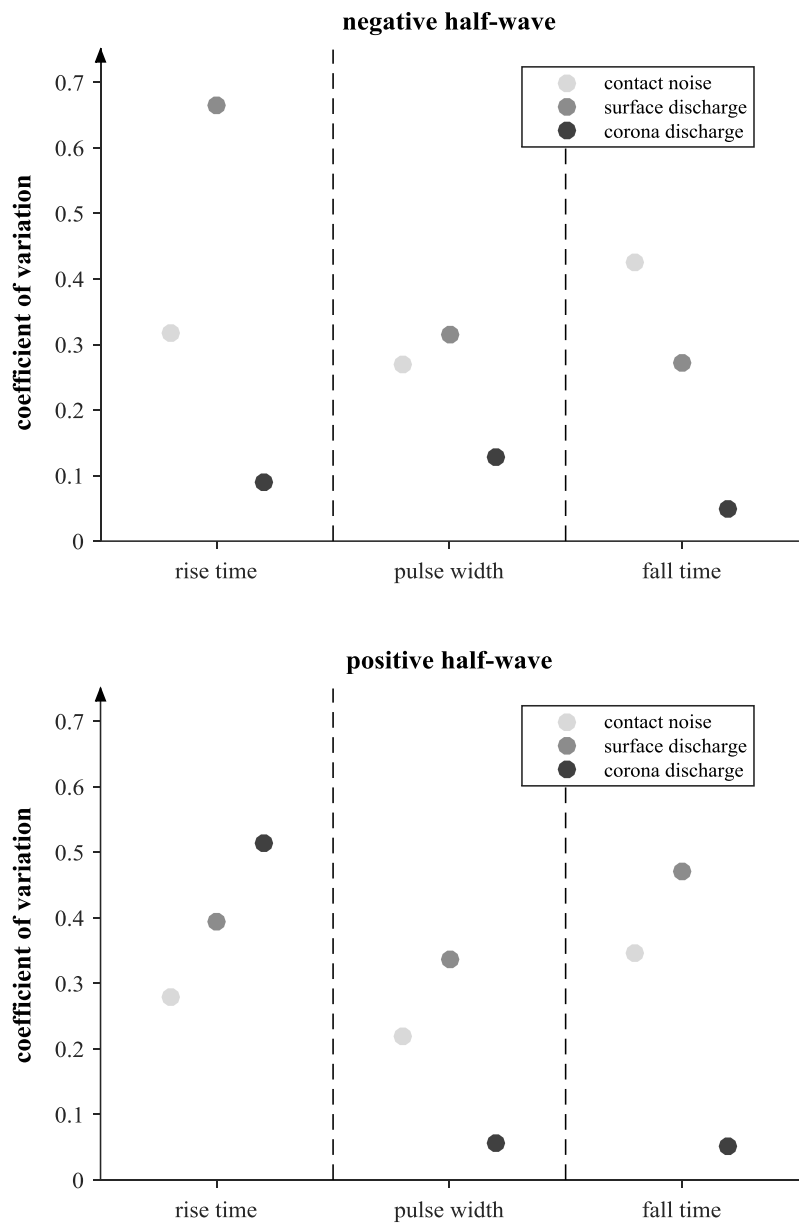

Fig. 8. Coefficients of variation for the pulse shape parameters rise time, pulse width and fall time of contact noise, surface discharges and corona discharges in ambient air occurring in the negative (top) and the positive (bottom) halfwave of the test voltage.

shape parameters reach larger values in the case of corona discharges occurring in ambient air. This is especially noticeable for the pulse width and the fall time. The difference is particularly clear for the fall time of discharges occurring in the negative half-wave. Whereas the determined values for negative corona discharges in ambient air are in the range of $77 \mathrm{~ns}$ to $98 \mathrm{~ns}$, those for negative corona discharges in oxygen are in the range of $13 \mathrm{~ns}$ to $21 \mathrm{~ns}$. Responsible for this behaviour is the different electronegativity and the associated electron attachment of the investigated gaseous insulating media. If the electronegativity increases, more electrons are converted to negative ions and the discharge process can be quenched in a lower time span [10]. Due to the fact that the electronegativity of oxygen is higher than the electronegativity of ambient air, the pulse shape parameters reach smaller values in the case of oxygen.

An overlapping of the location and the range of the confidence intervals is not determined. As a consequence it can be noted that the time domain analysis of corona discharges is suited to differentiate between discharges occurring in ambient air and oxygen for the chosen test conditions. 

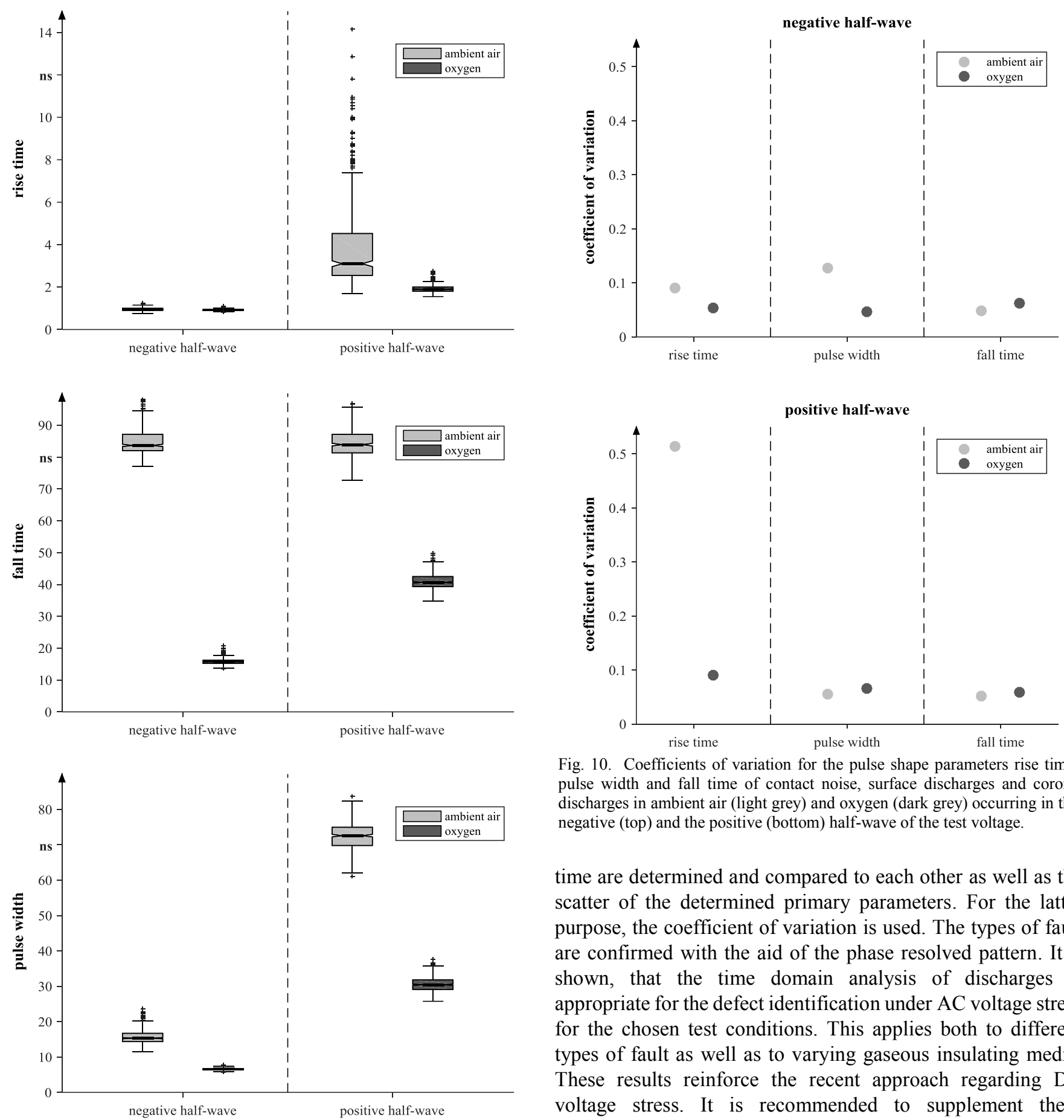

Fig. 9. Box plots for the pulse shape parameters rise time (top), pulse width (centre) and fall time (bottom) of corona discharges in ambient air (light grey) and oxygen (dark grey) occurring in the negative (left) and the positive (right) half-wave of the test voltage.

\section{CONCLUSION}

The pulse shapes of discharges caused by different types of fault in an optimised measuring cell are captured with an oscilloscope. For corona discharges the insulating medium is varied by using ambient air and oxygen. To analyse the pulse shapes, the primary parameters rise time, pulse width and fall

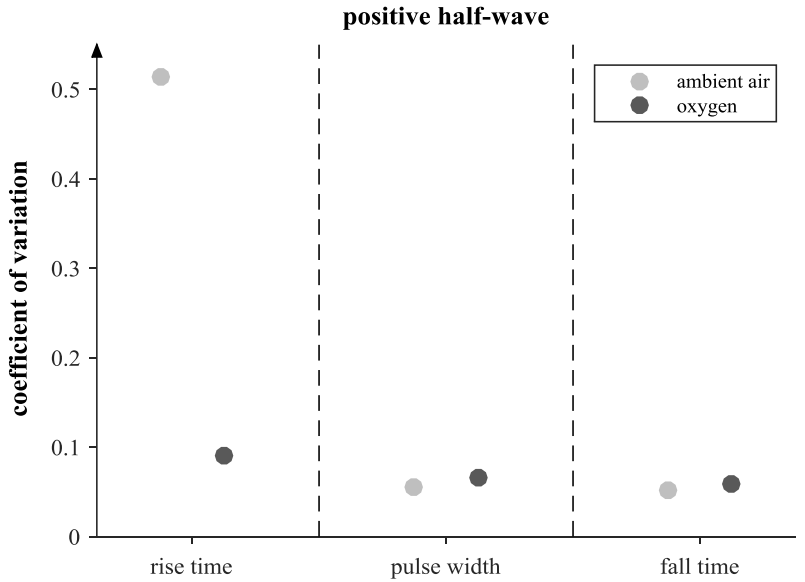

Fig. 10. Coefficients of variation for the pulse shape parameters rise time, pulse width and fall time of contact noise, surface discharges and corona discharges in ambient air (light grey) and oxygen (dark grey) occurring in the negative (top) and the positive (bottom) half-wave of the test voltage.

time are determined and compared to each other as well as the scatter of the determined primary parameters. For the latter purpose, the coefficient of variation is used. The types of fault are confirmed with the aid of the phase resolved pattern. It is shown, that the time domain analysis of discharges is appropriate for the defect identification under AC voltage stress for the chosen test conditions. This applies both to different types of fault as well as to varying gaseous insulating media. These results reinforce the recent approach regarding DC voltage stress. It is recommended to supplement these investigations through complementary research focussing on liquid insulating media and internal discharges. Furthermore it is recommend to investigate whether a distinction of different types of fault is still possible if the test conditions, such as pressure, voltage level and stress duration are varied. 


\section{REFERENCES}

[1] IEC 60270:2000/BS EN 60270:2001 "High-Voltage Test Techniques Partial Discharge Measurements".

[2] D. König and Y. N. Rao, "Partial discharges in electrical power apparatus", Berlin: VDE-Verlag, 1993.

[3] U. Fromm, "Interpretation of Partial Discharges at dc voltages", IEEE Transactions on Dielectrics and Electrical Insulation Vol. 2, No. 5, October 1995.

[4] P. Morshuis, "Assessment of dielectric degradation by ultrawide-band PD detection", IEEE Transactions on Dielectrics and Electrical Insulation Vol. 2, No. 5, October 1995.

[5] L.B. Loeb, "Electrical coronas - Their basic physical mechanism", Berkeley and Los Angeles: University of California Press, 1965.

[6] R. Morrow, "Theory of negative corona in oxygen", Physical Review A Vol. 32, No. 3, September 1985.

[7] D. A. Scott and G. N. Haddad, "Negative point-to-plane corona pulses in oxygen", Journal of Physics D: Applied Physics 19, 1986.

[8] M. Cernak, T. Hosokawa and I. Odrobina, "Experimental confirmation of positive-streamer-like mechanism for negative corona current pulse rise", Journal of Physics D: Applied Physics 26, 1993.

[9] X. Liu, S. A. Sebo, D. G. Kasten, D. L. Schweickart and D. F. Grosjean, „Partial discharge sub-atmospheric pressures - Methods of analysis of experimental results", Annual Report: Conference on Electrical Insulation and Dielectric Phenomena, 2007.

[10] H. Okubo, N. Hayakawa and A. Matsushita, "The relationship between partial discharge current pulse waveforms and physical mechanisms", IEEE Electrical Insulation Magazine Vol. 18, No. 3, 2002.

[11] H. Okubo and N. Hayakawa, "A novel technique for partial discharge and breakdown investigation based on current pulse waveform analysis", IEEE Transactions on Dielectrics and Electrical Insulation Vol. 12, No. 4, August 2005.

[12] J. M. Wetzer and P. C. T. van der Laan, „Prebreakdown Currents - Basic Interpretation and Time-resolved measurements", IEEE Transactions on Electrcial Insulation Vol. 24, No. 2, April 1989.

[13] T. Brosche, W. Hiller, E. Fauser and W. Pfeiffer, "Novel Characterization of PD Signals by real-time measurements of pulse parameters", IEEE Transactions on Dielectrics and Electrical Insulation Vol. 6, No. 1, February 1999.

[14] Z. Zheng and K. Tan, "Comparison of feature extraction methods in partial discharge waveform recognition", Annual Report: Conference on Electrical Insulation and Dielectric Phenomena, 2001.
[15] X. Li, G. Wu, X. Zhang and S. Bian, „Partial discharge pulse shape detection and analysis under dc condition", Electrical Insulation Conference and Electrical Manufacturing, October 2007.

[16] Suwarno and T. Mizutani, "Diagnosis of insulation conditions Interpretation pf partial discharges from $\Phi$-q-n pattern, pulse-sequence and pulse waveform", International Conference on Condition Monitoring and Diagnosis, Beijing/China, April 2008.

[17] S. Wenrong, L. Junhao, Y. Peng and L. Yanming, "Digital Detection, Grouping and Classifiaction of Partial Discharge Signals at DC Voltage", IEEE Transaction on Dielectrics and Electrical Insulation Vol. 15, No. 6, December 2008.

[18] T. Klueter, J. Wulff, F. Jenau and D. Wienold, "Evaluation of Surfaceand Corona Discharges at DC Voltage", $13^{\text {th }}$ International Conference on Environment and Electrical Engineering, Wroclaw/Poland, November 2013.

[19] T. Klueter, J. Wulff and F. Jenau, "Time Domain Analysis of Partial Discharges at DC Voltage in Air and Insulation Oil “, $12^{\text {th }}$ International Conference on Environment and Electrical Engineering, Wroclaw/Poland, May 2013.

[20] T. Klueter, J. Wulff and F. Jenau, "Measurement and Statistical Analysis of Partial Discharges at DC Voltage", $48^{\text {th }}$ International Universities' Power Engineering Conference UPEC 2013, Dublin/Ireland.

[21] T. Vogt, "Teilentladungsdiagnose bei Gleichspannung", Dissertation Technische Universität Dortmund, 2015.

[22] M. Kurrat and D. Peier, "Wideband measurement of partial discharges for fundamental diagnostics", $7^{\text {th }}$ International Symposium on High Voltage Engineering, Dresden/Germany, August 1991.

[23] F. H. Kreuger, "Discharge Detection in High Voltage Equipment", London: Heywood, 1964.

[24] D. Borneburg, "Bewertung von Hochspannungsisolatoren als Quelle hochfrequenter Störfelder“, Dissertation Universität Dortmund, 2003.

[25] J. M. Chambers, W. S. Cleveland, B. Kleiner and P. A. Tukey, "Graphical Methods for Data Analysis", Belmont: Wadsworth International Group, 1983.

[26] P. H. F. Morshuis, "Partial discharge mechanisms", Delft University of Technology, 1993.

[27] A. Schwab, "Über die Anstiegszeiten von Koronaentladungsimpulsen in einer Spitze-Platte Funkenstrecke“, Dissertation Technische Hochschule Karlsruhe, 1963. 